\title{
Why I Retweet? Exploring User's Perspective on Decision-Making of Information Spreading during Disasters
}

\author{
Nor Athiyah Abdullah \\ Universiti Sains \\ Malaysia, Malaysia \\ athiyah@usm.my
}

\author{
Dai Nishioka \\ Iwate Prefectural \\ University, Japan \\ nishi_d@iwate-pu.ac.jp
}

\author{
Yuko Tanaka \\ Nagoya Institute of \\ Technology, Japan \\ tanaka.yuko@nitech.ac.jp
}

\author{
Yuko Murayama \\ Tsuda College, Japan \\ murayama@tsuda.ac.jp
}

\begin{abstract}
The extensive use of social media during disasters raises an important issue concerning use of social media to spread information, including misinformation. This study explores the underlying behavioral context of disaster information sharing by Twitter users. We conducted a web survey with 999 respondents in Japan to determine what makes people retweet disaster information in disaster situations. As a result of factor analysis, four factors were identified from 36 questions, namely: 1) Willingness to provide relevant and updated information because the information is believable, 2) Want people to know the information they perceive as important, 3) "Retweeter" subjective feelings and interests, and 4) Want to get feedback and alert other people. The results suggest that two of the factors influenced different groups of people in the community differently; however, everybody can play their role to reduce the negative impact of social media used for future disaster. Based on the findings, we discuss practical and design implications of social media use during disasters.
\end{abstract}

\section{Introduction}

Twitter is a microblogging service which allows everyone to generate and share ideas and information instantly without barriers [1]. Twitter enables registered users to broadcast short posts up to 140 characters called tweets, and they can spread other users' tweets by retweeting. Twitter serves many roles, for example as a social sensor to detect earthquake events [2], to facilitate the transmission of rumors [3], to influence social and political awareness [4], to act as a news medium [5] and also to coordinate humanitarian relief during disaster responses [6]. Several studies in the literature discuss the effectiveness of social media in providing updated information and engaging with citizens during disasters such as during the Victorian bushfire [7], the Haitian Earthquake [6], The Great East Japan Earthquake $[8,9,10]$ and Hurricane Sandy [11].

However, the use of social media during disasters raises an important issue concerning information credibility $[19,20,21]$ as Twitter also has the potential to facilitate misinformation and rumor transmission in emergency situations [3, 8, 12, 13]. Misinformation may not only cause a delay in response and effort for emergency management rescue, it also affects the public: people want to know how they should prepare and react to the ambiguous situation happening around them. Manoj and Baker [14] state challenges to designing effective communication systems for crisis situations. Of relevance for this paper is the sociological aspect, where there is a need to understand people's models of human activity and communication behavior. Another study highlights the need to investigate user behavior towards crisis information dissemination from a psychological viewpoint [15]. Thus, this research is motivated by the need to understand user information diffusion behavior using during disasters using Twitter.

There are few research from psychology viewpoint investigate the relationship between anxiety, importance, distance and feelings with rumor transmission and crisis-information sharing behavior in disaster situation [13,15,30]. Meanwhile, previous studies on retweeting behavior mainly focus on the structure of the social network and the information topic and content $[16,17,18,22]$. Since most investigations of retweeting behavior comes directly from social network data, our research contribution uses self-reports and directly taps into the issue of motivation.

Because everyone is capable of spreading information in social media, we want to investigate from retweeter's perspective, what makes them, in a disaster situation, retweet disaster information they read from Twitter. In our study, we focused on the scenario when a Twitter user reads disaster-related information. We investigated the factors that influence 
the user's decision to spread by retweet this information. First, we conducted an exploratory study by brainstorming to gather ideas from targeted respondents and then we created the preliminary questionnaire for the rest of the study. Next, we conducted a pilot study to test and improve the questionnaire before we distributed it to a larger sample, in the form of a web survey. The principal aim was to understand the individual information spreading behavior which may cause information, including misinformation to circulate in Twitter during disasters.

In this paper, we present the results and findings of the web survey. Using exploratory factor analysis we extracted factors related to individual motivation to spread disaster information from the entire sample. Next, we conducted further analysis to discover whether the factors correlate to different categories of people in the community, such as the disaster victims, family or friends are affected, volunteers, and the general public. Based on the findings, we discuss practical and design implication on how to utilize social media effectively during disasters.

In this research, our goal is to help users make better decisions with regard to information spreading behavior in social media. Better understanding of why people choose to spread information in social media is helpful to improve the usefulness of social media as an important disaster communication tool. Therefore, this research aim to answer the research questions as follows:

RQ1: In disaster situations, from the retweeter perspective, why do people decide to retweet disaster information?

RQ2: Are there any differences in motivation to spread disaster information among different groups of people in the community?

The rest of the paper is organized as follows. Section 2 discusses the related work. In section 3 we describe the survey. Section 4 presents the analysis of results. Section 5 contains the discussion of our findings and the limitations of the current work along with future work recommendations. Finally, we conclude our work in section 6 .

\section{Related Work}

\subsection{Social Media during disasters}

In recent years, several studies focused on the utilization of social media for mass collaboration in response and rescue for emergency management professionals during emergencies [11, 19, 20]. Crisis informatics research views emergency professionals as an expanded social system that includes the dissemination of information between and within official and public channels and citizens [23]. Social media serve as new routes for information flows and also as channels to provide information during disasters for those in need such as the survivors, emergency responders, volunteers and also the general public [23]. Information received from citizens via social media proved to be useful, especially at the area level, in coordinating humanitarian relief after the 2010 Haiti earthquake [6]. During Hurricane Sandy in 2012, the US government used Twitter for information exchange with citizens in disaster-related preparation, response and recovery stages [21]. There is no doubt that social media have become one of the most dependable disaster communication tools for citizens and authorities to engage one another during disasters. However, during disasters, there is much ambiguity, the need for update information is often crucial, so people tend to accept any information which helps them to make sense of the situation, including unverified information or rumor. On Twitter, information can continuously change from correct to incorrect due to retweeting timing [24].

Nowadays, with social media, everybody can generate and disseminate information because they are the real first respondents in the event [25]. Although the information from citizen is helpful for disaster response, Raue [20] says that about $43 \%$ of emergency management professionals agreed that there is too much misleading information on social media. Misleading information may not only cause delays in response and rescue efforts by emergency professionals, but may also affect the public who wants to know how they should prepare and react to the ambiguous and vague situation happening around them.

On the other hand social media is also an effective communication tool for professionals to engage with the public, and to verify or counter rumors. Authorities created official Twitter accounts to engage with citizens during 2012 Hurricane Sandy [21], 2009 Victorian bushfires [7] and 2011 Great East Japan Earthquake [28]. There are also Twitter accounts (for example, @IsTwitWrong) created by the public to criticize and combat fake images spreading in Twitter. These uses show that Twitter is also a beneficial tool to combat misinformation from spreading, not only for authorities or official organizations to make announcement or provide information, but also as a platform for the public to voluntarily cooperate and contribute their efforts in reducing fake news in social media. However, rumor spreading will never go away. Some individuals might keep spreading rumors even as other people try to prevent them using criticism, and other rumor control techniques [27]. In Japan, Twitter was listed as the top form of social media used to gather disaster-related information after the 2011 Great 
East Japan Earthquake $[10,28]$. After the mega earthquake strike on March 11, Twitter was flooded with various information reporting self-experience, warning, fact, safety status and even rumor and hoax messages [4,9,29]. According to analysis by Fukushima [4], most of the tweets during The Great East Japan Earthquake were accurate and highly reliable, but there was also noise, particularly in the disaster affected area.

\subsection{Human behavior during disasters}

Empirical findings by Schulze et al [30] reported that majority of people claimed that they would react rational and will help each other in disaster situations. Disaster research generally agrees that people tend to act prosocially and with altruism during disasters [31]. Although the use of Twitter has several issues, Twitter supports the prosocial role during disasters, and is also a basis to build social capital among people who are lightly affected by the disaster [26]. Disaster scenarios may lead to solidarity where people share a sense of danger and fate, and act selflessly even among strangers [31].

On information sharing behavior, psychological research has found that when people have negative feelings such as anger, nervousness or worry, they tend to spread crisis information [13]. Tanaka [27] explored the relationship between perceived accuracy, importance and anxiety on rumor spreading behavior in social media. Li et. al [32] state that the ease of processing, or fluency of the information influence people's decision to spread the information. A recent study by $\mathrm{Li}$ [33] revealed that the retweet count, influenced people likelihood to share the tweets from an individual Twitter account.

On the other hand, research in the emergency management field indicates that judgment and decision-making of emergency managers under stress is influenced by the analytical or cognitive factors such as knowledge that one possesses, along with experience and emotional factors [34]. Dugdale et. al [6] state that the emotional state of citizens affected texting behavior during the 2010 Haiti Earthquake. Gupta et al. [11] indicate that in case of crisis, people often retweet things that they find in twitter searches or trending topics, regardless of whether they follow the user or not. With citizen participation in supplying disaster information through their own social networks, trustworthiness, information overload and privacy issues raised the barrier for emergency managers in utilizing social media during emergencies [35].

In general retweeting behavior, not particularly in disaster situations, research suggests that by retweeting people want to be in a conversation, to share relevant information and sometimes to selfishly seek attention $[17,36]$. People retweet information they believe will capture their follower's interest and thereby acquire a chance to get retweeted [36]. Mackassy and Michelson [18] indicate that a content-based model, taking into account homophily in terms of the user profile and tweet topic, is better explains why people retweet information. Most of the work highlights that why people retweet is based on what they retweet. During emergencies, people often retweet information that they feel is valuable and important for others to know, even upon the request of a stranger [37,38].

Investigation of why people decide to spread disaster information at the time of the disaster and their motivations to retweet are still lacking. At a time when people need information, with a bundle of information available from social media, one needs to decide to accept or not the available information. Retweet practice is related to the motivations of users who decide to retweet [36]. Therefore, in this study, we aim to explore and understand from retweeters' perspectives, what is their motivation to spread disaster information. To the best of our knowledge, there is no related work to answer our research questions. Therefore, we conducted an exploratory study by brainstorming and created a questionnaire.

\subsection{Create the Questionnaire}

Researches often design the questionnaires using various techniques such as brainstorming [39], adoption from the literature [40], definition of variables from the literature [32] and conducting interviews [41]. Rashtian et. al [41] conducted interviews in an exploratory study to understand users' befriending behavior on Facebook and to explore factors that influence their decisions. They conducted the exploratory study because there were no related works that support their research question. Another study conducted a questionnaire survey based on question items adopted from several previous studies on related topics [40]. Research from a psychological viewpoint on the use of social media created questionnaire items based on the definition of proposed variables from the literature [13,27]. Brainstorming can produce holistic and creative ideas [42]. That is why, in this research, we chose the brainstorming technique to gather ideas which facilitated us to produce the new questionnaire. Instead of personally interviewing each individual at a different time, we used brainstorming to gather ideas from targeted respondents, which are the social media users. 


\section{The Survey}

The Great East Japan Earthquake with magnitude 9.0 on March 11,2011 was the most catastrophic event ever to hit Japan in the new century. It caused severe damage to the northern coast of the main island in Japan, especially in the Tohoku region, the Iwate, Miyagi and Fukushima prefectures. The disaster triggered a tsunami and caused more global problems because of the Fukushima nuclear radiation disaster. Floods, landslides, fires, building and infrastructure damage all occurred, and electricity, telecommunication and transport suffered severe disruptions especially in the disaster area.

In our survey, we collected respondents' information which refers to their role during the 2011 Great East Japan Earthquake. Disaster victims were the ones who were directly affected during the disaster, especially in the Tohoku region. Some respondents might not be in the disaster area, but their family or close friends were affected, for example, people staying in Tokyo, but whose family or friends lived in the disaster area. The volunteers or supporters are from the areas which were not severely damaged by the disaster, but they were close to the disaster area. After the disaster, these volunteers and supporter went to the disaster area to provide technical support and other help in the affected area. The public includes people where were not directly affected; they live far from the disaster area. With the use of Twitter as an important medium to communicate and disseminate information to people from organizations and citizens, we are interested to know, among the citizens, are there any difference in their motivation to spread disaster information?

\subsection{Participants}

By using the web survey company service, the web survey was conducted from $27^{\text {th }}$ to $31^{\text {st }}$ of July 2015, with 1032 response. However, 33 of them were excluded because they answered all questions with the same answer (Standard Deviation $=0$ ), which in turn could lead to bias in response. Therefore, 999 valid responds remain in the analysis. The mean age of the respondents is 35.91 years old. Before the respondents answered the questionnaire, they were screened with three conditions. First, they must be a Twitter user. Second, they must be an information spreader, which means they have ever retweeted information from Twitter, and third, they must have utilized Twitter to get disaster information. We used the case of the 2011 Great East Japan Earthquake as an example of a catastrophic event in Japan, focused on Twitter because during the disaster, Japanese people used Twitter more than other social media. We also collected the respondent's role, whether they are a disaster victim, their family or friends were affected, they were a volunteer or supporter, or they were the public, and were not directly affected by the disaster.

There are 8 regions and 47 prefectures (similar to states) in Japan. The 8 regions are Hokkaido, Tohoku, Kanto, Chubu, Kinki, Chugoku, Shikoku, and Kyushu. The most affected region during the disaster was the Tohoku region. The respondents in this survey are from all regions in Japan, and $16.4 \%$ of them are from the Tohoku region. Table 1 shows the demographic information of the respondents in the web survey.

Table 1. Demographic information

\begin{tabular}{|lll|}
\hline Gender & Male & $45.8 \%$ \\
& Female & $54.2 \%$ \\
\hline Age & $20-29$ & $39.4 \%$ \\
& $30-39$ & $28.4 \%$ \\
& $40-49$ & $20.6 \%$ \\
& $50-59$ & $11.7 \%$ \\
& 60 and above & $3.4 \%$ \\
\hline Group & Victim & $24.8 \%$ \\
& Family or friends were & $25.2 \%$ \\
& affected & \\
& Volunteer or supporter & $25.5 \%$ \\
& Public (not directly affected) & $24.4 \%$ \\
\hline Area & Hokkaido & $4.1 \%$ \\
& Tohoku & $16.4 \%$ \\
& Kanto & $45.8 \%$ \\
& Chubu & $9.9 \%$ \\
& Kinki & $14.6 \%$ \\
& Chugoku & $2.9 \%$ \\
& Shikoku & $1.4 \%$ \\
& Kyushu & $4.8 \%$ \\
\hline
\end{tabular}

\subsection{Questionnaire Design and Analysis}

The questionnaire was developed by brainstorming technique with 10 participants and following the procedure [43]. The participation in brainstorming was voluntary, and we did not provide any incentive to the participants. The purpose of brainstorming was to gather ideas from targeted respondents to help us create the questionnaire. The ideas were then sorted and categorized using the KJ method so that we could check and eliminate redundant points. We tested the questionnaire developed in a pilot study $(n=57)$ and corrected the questionnaire before distributing it in the web survey. The survey was originally distributed in Japanese language, but in this paper, we report it in English as in 
the appendix. The main part of the survey consists of 38 question items on 7-point Likert scale (1=strongly disagree, 7=strongly agree) regarding why the user retweets disaster information in a disaster situation.

For the analysis part, we performed exploratory factor analyses (EFA) with the maximum likelihood method and promax rotation. Similar to other studies $[43,39]$, we performed EFA to explore and identify factors that influenced individual decision making to spread disaster information. Then, we performed Cronbach alpha test to measure the internal consistency and how closely related the items in a group were.

\section{Results}

\subsection{Factor Analysis}

For the first step before the factor analysis, we gathered descriptive statistics on all 38 question items and analyzed whether there are floor and ceiling effect questions, high skewness and kurtosis and problems with Cronbach alpha value. We do not have a problem with skewness and kurtosis value, so we can assume that the data is normally distributed. Out of 38 question items analyzed, there is a 1 question (Q5) with floor effect. It means that most of the respondents disagree with the statement. Since we want to extract factors influencing users' decisions to spread disaster information, we excluded this item from the analysis. However, our analysis shows that minority number, $10.5 \%$ of the respondents stated they agree with this question item's statement (Q5). The table in the appendix shows the descriptive statistics with mean and standard deviation values for all question items. Next, we conducted an exploratory factor analysis (EFA) with maximum likelihood method using SPSS 21 on 37 question items. We used the scree plot method to extract the factors structure is by using the scree plot. Factor analysis with the maximumlikelihood method and the promax rotation identified four factors. However, one question (Q27) is closely correlated with two factors (factor 1 and factor 2) with difference of 0.012 . So, we eliminate this question item.

As a result, 36 question items remained for EFA. The cumulative value for the factors are $59.997 \%$. The cumulative value describes how much the factors explained all the question items. Table 2 below shows the pattern matrix with factor loadings for each factor.

Table 2. Factor Pattern Matrix

\begin{tabular}{|l|r|r|r|r|}
\hline $\begin{array}{l}\text { Quest } \\
\text { ion } \\
\text { items }\end{array}$ & Factor 1 & Factor 2 & Factor 3 & Factor 4 \\
\hline Q22 & .707 & .171 & -.048 & -.044 \\
\hline
\end{tabular}

\begin{tabular}{|c|c|c|c|c|}
\hline Q23 & .692 & .113 & .017 & -.052 \\
\hline Q34 & .682 & .002 & .074 & .080 \\
\hline Q24 & .667 & .240 & -.086 & -.019 \\
\hline Q32 & .662 & .054 & -.097 & .177 \\
\hline Q28 & .640 & .341 & -.052 & -.048 \\
\hline Q33 & .638 & -.065 & .022 & .284 \\
\hline Q21 & .629 & -.050 & .167 & .019 \\
\hline Q35 & .621 & .308 & -.009 & -.061 \\
\hline Q26 & .597 & .271 & -.017 & .038 \\
\hline Q25 & .596 & .323 & -.035 & -.014 \\
\hline Q36 & .585 & -.062 & .364 & -.056 \\
\hline Q29 & .576 & .247 & .004 & .028 \\
\hline Q38 & .515 & .143 & .195 & -.073 \\
\hline Q30 & .500 & -.106 & .363 & .111 \\
\hline Q13 & .225 & .798 & -.197 & -.155 \\
\hline Q10 & .017 & .761 & -.111 & -.016 \\
\hline Q11 & .085 & .702 & .005 & .089 \\
\hline Q7 & -.069 & .689 & .259 & -.075 \\
\hline Q9 & -.013 & .656 & .095 & .143 \\
\hline Q14 & .141 & .512 & -.144 & .217 \\
\hline Q2 & -.129 & .497 & .309 & .119 \\
\hline Q12 & .245 & .460 & -.033 & .171 \\
\hline Q19 & .251 & .459 & .025 & .101 \\
\hline Q16 & .175 & .377 & .249 & .072 \\
\hline Q4 & -.076 & -.016 & .799 & .101 \\
\hline Q1 & -.015 & .278 & .606 & -.060 \\
\hline Q6 & -.046 & -.087 & .592 & .273 \\
\hline Q37 & .387 & -.289 & .530 & .028 \\
\hline Q3 & .110 & .406 & .435 & -.244 \\
\hline Q20 & .282 & .063 & .372 & .184 \\
\hline Q18 & .005 & -.039 & .037 & .886 \\
\hline Q17 & .132 & .003 & .089 & .679 \\
\hline Q31 & .366 & -.178 & .032 & .648 \\
\hline Q8 & -.231 & .271 & .212 & .553 \\
\hline Q15 & .076 & .271 & .010 & .490 \\
\hline $\begin{array}{l}\text { Cumu } \\
\text { lative } \\
\%\end{array}$ & $49.516 \%$ & $55.060 \%$ & $57.934 \%$ & $59.997 \%$ \\
\hline $\begin{array}{l}\text { Factor } \\
\text { correl } \\
\text { ation } \\
\text { matrix } \\
: \text { F1 }\end{array}$ & 1.00 & .713 & .620 & .676 \\
\hline $\mathrm{F} 2$ & .713 & 1.00 & .524 & .507 \\
\hline F3 & .620 & .524 & 1.00 & .660 \\
\hline $\mathrm{F} 4$ & .676 & .507 & .660 & 1.00 \\
\hline
\end{tabular}

Factor analysis helps us to answer our first research question as follows:

RQ1: In a disaster situation, from the retweeter perspective, why do people decide to retweet disaster information?

We identified the factors as factors related to user's decision making to spread the disaster information during disasters as follows: 
Factor 1: Willingness to provide relevant and updated information because the information is believable.

This factor consists of 15 items regarding individual acts to collect and provide updated related information they received from Twitter for oneself and their followers. It includes information from people they trust, information with proof (picture or video), and early information which is helpful such as early information for safety status check.

Factor 2: Want people to know the information they perceived as important.

This factor consists of 10 items related to individual evaluation that the information is crucially important and should be spread. For example, the information came from credible source, warning information, and information that the retweeter has knowledge of and they believe it is important for other people to know.

Factor 3: 'Retweeter' subjective feeling and interest.

This factor consists of 6 items related to the retweeter's decision to retweet because the information captured their interest and they felt excited to share about the unusual situation occuring during the disaster. It also subjects to retweeter's Anshin (sense of security) or comfortable feeling in the information after they saw high number of retweets.

Factor 4 : Want to get feedback and alert other people.

This factor consists of 5 items regarding individuals' decision to retweet because they want to get response and feedback from the audience, and also to remind other people so that they are alert about it.

\subsection{Comparison of groups based on factor score}

Further analysis can be done using factor scores to identify groups of participants who score highly on a particular factor [45]. Next, we attempted to determine if there are significance differences of response between 4 groups of respondents: 1) Group 1: The disaster victims, 2) Group 2: family or friends were affected, 3) Group 3: volunteers or supporters, and 4) Group 4: the public.

We seek to answer the second research question:

RQ2: Are there any difference in motivation to spread disaster information among different groups of people in the community?

Based on the ANOVA analysis of Tukey's test, only factor 3 and factor 4 shows significantly different mean, among groups of respondents $(\mathrm{p}<.05)$. For factor 1 and 2 there is no significant difference of mean among these 4 groups. Group 3, which is the volunteer/supporter group has the highest mean on factor 3 ('Retweeter' subjective feeling and interest). The reason why could be related to solidarity and to getting involved on trending topics which occur during disasters. For factor 4 (Want to get feedback and alert other people), compared to other groups, group 4 which is the public scores the lowest mean. One of the reasons why is because the public are the ones who are not directly affected by the disaster, nor are their close family and friends affected, so their main reason to retweet during disasters is not to get feedback from the audience.

\subsection{Reliability Test}

According to Cronbach [46], "any research based on measurement must be concerned with the accuracy or dependability or, as we usually call it, reliability of measurement". Cronbach alpha is a measure of internal consistency or reliability, which means, how closely related a set of items are as a group. For reliability analysis, we should run separate reliability analysis for all subscales (factor) emerged from the questionnaire. For the reliability measure, the Cronbach's coefficient alpha for each factor subscale factor 1 , factor 2 , factor 3 , and factor 4 are $0.956,0.917,0.842$, and 0.888 respectively. The Kaiser-Meyer-Olkin (KMO) value is 0.975. In sum, according to Field [45], our results yield to reliable factor analysis of the criteria:

1) Excellent sample size $(n=999)$, the good sample size is at least 300 respondents.

2) The KMO value close to 1 .

3) Reliability test, the Cronbach coefficient alpha value is more than 0.7 for each subscale.

\section{Discussion}

The findings help us to gain insight into factors that may influence individuals, as potential information spreaders on their decisions to spread disaster information using Twitter as a disaster communication tool. The first factor, "Willingness to provide relevant and updated information because the information is believable", refers to collecting and providing updated related information they received from Twitter for themselves and their followers. It includes the information from people they trust, information with proof (picture or video), and early information which is helpful such as early information for safety status check. Similarly, previous studies also highlight content relevance as the reason for retweeting during disasters [38].

The second factor, "Want people to know the information they perceived as important" is related to 
individual evaluation as the information is crucially important and should be spread. For example, the information comes from a credible source, is warning information, or is information that retweeter has knowledge of it and they believe it is important for other people to know. This reason is consistent with Lee et al. [38] on the trustworthiness of the tweet content as the reason of why people retweet. Tanaka et al. [27] concluded that individual perception of accuracy influences individuals' decision to spread rumors, which is unverified information.

Emotions influence information sharing during disasters [13]. One conclusion based on the current results is that people also spread disaster information based on their subjective feeling. The third factor "'Retweeter's subjective feeling and interest" is related to retweeter's decision to retweet because the information captures their interest and they feel excited to share about the unusual situation topic occur during a disaster. It also subjects to retweeter's Anshin (sense of security) or comfortable feeling in the information after they saw high number of retweets. As stated by Gupta et al [11] the act of spreading trending topics influences why people spread tweets during disasters. Although we cannot tell from our dataset in what way the high number of retweets influenced decision to retweet, another recent study by Li and Sakamoto [33] found that the retweet count, influenced people's likelihood to share tweets from an individual Twitter account.

The fourth factor, "Want to get feedback and alert other people" regards the individuals retweeting because they want to get response and feedback from the audience, and also to remind other people so that they are alert about it. This factor reflects the prosocial behavior [31] that leads to solidarity and selfless acts even among strangers in crisis situations. Boyd et al [17] writing about general retweeting behavior, not particularly during disasters, indicates that one of the reasons why people retweet is in order to be in a conversation. Similarly, during disasters, when people need the accurate information, by retweeting, they can receive instant feedback from followers regarding the information they spread, for example if it is an inaccurate information or rumor.

\subsection{Practical and Design Implications}

The results presented in this work may help users make better decisions with regard to information spreading using Twitter during disasters. People may have good intentions to help, by retweeting for awareness those who are affected by the disaster; however, in some cases, they might also unintentionally contribute to the circulation of false rumors in an already tense situation. Citizen actions when confronted with a disaster can be divided into two types: first, intuitive and emotion based, and second, analytical, based on reasoning [47]. We suggest that individuals, especially those who are not directly affected by the disaster, such as the volunteers or the general public, can play a role to help minimizing the spread of unverified information by applying analytical thinking, and at least by looking at how other people reply to that particular information. We might not be able to decide whether every information we received is true or not, since different people may have different knowledge and prior experience. On Twitter, we can look at other people's responses in the form of their reply to that particular tweet. Screening based on other people responses and opinions, helps us to think twice and make better decisions. Individuals can help reduce information overload in social media by spreading credible information with a reliable source. Ambiguity of the information's source is one attribute of rumors [48].

On the flip side, as disaster communication is enhanced by the use of social media, authorities and organization should utilize Twitter to provide immediate and timely information to citizens. What people need most during disasters is updated information as they want to know what has happened around them. According to Shibutani [48], rumors are generated if the demand for news is high, but the information supply is low. If the supply and demand of news are balanced, then the rumors disappear. In this case, governments' and organizations' official Twitter accounts could help reduce the generation and spreading of unverified information by providing immediate, reliable information to citizens.

The designers of social media may consider creating a disaster mode for their applications. Let us take a look at the current Twitter interface design for an example. Current functions in Twitter allow people to "favorite" the tweet, perform retweet instantly or add their own opinion on the tweet by quote and retweet. The current Twitter design only allows users to report spam tweets, sensitive or harmful tweets, and non-interested tweet. A disaster mode could provide a report button for users to alert for unverified information or tweets with an unclear source of information so that other users are aware of the information truthfulness risk. Such attributes would be useful for users to make better decisions about further spreading the information.

\subsection{Limitations and Future Work}


This study has several limitations. First, as with other research regarding users' behavior, the results that we presented indicate users' after-the-fact, selfreported motivations, and they may not reflect real behavior during an actual disaster. Second, the current study's focus is on the Twitter environment and the respondents are all from Japan. The current results may or may not generalize to other samples of respondents, or to other types of social media such as Facebook.

Detailed attributes of the retweeter and the original author (eg: location, type of Twitter user) at the time of the actual disaster may provide fruitful findings on how these 4 factors influenced peoples' decision to spread disaster information. Finally, our current research focus is generally on information spreading behavior, which may or may not include misinformation in the Twitter dataset. Future work should work on misinformation or rumor data as well.

\section{Concluding Remarks}

To understand the human information spreading behavior during disasters, we took the approach of conducting a user survey using a questionnaire developed from brainstorming with the target group, which are the social media users. Overall, four factors emerged to explain what motivates people to spread disaster information during disasters. The results of the exploratory factor analysis found that individuals spread disaster information because of: 1) Willingness to provide relevant and updated information because the information is believable, 2) Want people to know the information they perceived as important, 3) Retweeter' subjective feeling and interest, and 4) Want to get feedback and alert other people. Our results suggest that two of the factors are different among different groups of people in the community.

In conclusion, people spread disaster information mainly to help and fulfill other person's satisfaction, and also to fulfill their own satisfaction and needs. Since people will rely on social media for disaster communications, we believed that continued research in this area will contribute to an understanding of human behavior using these technologies in order to improve the design of social media to better prepare for future disasters.

\section{Acknowledgment}

Our deepest appreciation goes to Dr. Carl Hauser for the valuable comments and English editing of this paper. The project presented here was supported partly by a Disaster Response Project Research from Centre for Regional Policy and Studies, Iwate Prefectural University, Japan.

\section{References}

[1] Twitter, Inc.: About Twitter, Inc., 2015. https://about.twitter.com/company

[2] Sakaki, T., Okazaki, M., and Matsuo, Y.: Earthquake shakes Twitter users: real-time event detection by social sensors. Proc. of the 19th Int. Conference on WWW '10, ACM, NY, USA, 2010, pp. 851-860.

[3] Tanaka, Y., Sakamoto, Y., and Matsuka, T.: Transmission of Rumor and Criticism in Twitter after the Great Japan Earthquake. Annual Meeting of the Cognitive Science Society, 2012, pp. 2387-2392.

[4] Fukushima, Y., et.al: Macroanalysis of Microblogs: An Empirical Study of Communication Strategies on Twitter During Disasters and Elections. In: AAAI Spring Symposium Series, 2014.

[5] Kwak, H., Lee, C., Park, H., and Moon, S.: What is Twitter, a social network or a news media?. Proc. of the $19^{\text {th }}$ Int. Conf. on WWW '10, ACM, NY, USA, 2010, pp.591-600. [6] Dugdale, J., Van de Walle, B., and Koeppinghoff, C.: Social media and SMS in the haiti earthquake. Proc. of the $21^{\text {st }}$ Int. Conf. on WWW '12 Companion, ACM, NY, USA, 2012, pp. 713-714.

[7] UK Government : Using Social Media in Emergencies : Smart Practices, 2012.

https://www.gov.uk/government/uploads/system/uploads/atta chment_data/file/85946/Using-social-media-in-emergenciessmart-tips.pdf

[8] Peary, B.D.M., Shaw, R., and Takeuchi, Y.: Utilization of Social Media in the East Japan Earthquake and Tsunami and its Effectiveness. Journal of Natural Disaster Science 34(1), 2012, pp.3-18.

[9] Acar, A., and Muraki, Y.: Twitter for crisis communication: Lessons learned from Japan's tsunami disaster. Int. J. Web Based Communities, 2011, pp.392-402.

[10] Jung, J.: Social media use and goals after the Great East Japan Earthquake, First Monday, 17(8), 2012.

[11] Gupta, A., Lamba, H., Kumaraguru, P., and Indraprastha, A. J.: Faking Sandy: Characterizing and identifying fake images on Twitter during Hurricane Sandy. Proc. of the 22nd Int. Conf. on WWW'13 Companion, 2013, pp.729-736.

[12] Hagar, C.: Crisis informatics: Perspectives of trust -Is social media a mixed blessing? Student Research Journal, 2(2), Article 2, 2012.

[13] Chen, R., and Sakamoto, Y.: Perspective matters: Sharing of Crisis Information in Social Media. Proc. of the HICCS-46, 2013, pp.2033-2041.

[14] Manoj, B.S., and Baker, A.H.: Communication 
challenges in emergency response. Communications of the ACM, 2007, pp.51-53.

[15] Chen, R., and Sakamoto, Y.: Feelings and Perspective matter: Sharing of Crisis Information in Social Media. Proc. of the HICCS-47, 2014,pp. 1958-1967.

[16] Tinati, R., Carr, L., Hall, W., and Bentwood, J.: Identifying communicator roles in twitter. Proc. of the 21st Int. Conf. on WWW '12 Companion. ACM, NY, USA, 2012, pp.1161-1168.

[17] Boyd, D., Golder, S., and Lotan, G.: Tweet, Tweet, Retweet: Conversational aspects of Retweeting on Twitter. Proc. of the HICCS-43, 2010, pp.1-10.

[18] Macskassy, S. A., and Michelson, M.: Why Do People Retweet? Anti-Homoplihy Wins The Day!. Proc. of the Fifth International AAAI Conference on Weblogs and Social Media, 2011, pp.209-216.

[19] White, C., Plotnick, L., Kushma, J., Hiltz, S. R., and Turoff, M.: An online social network for emergency management. Int. J. Emergency Management, 2009, pp.69382.

[20] Raue, S., Azzopardi, L., and Johnson, C.W.: \#trapped!: Social media search system requirements for emergency management professionals. Proc. of the 36th Int. ACM SIGIR Conference on Research and Development in Information Retrieval, 2013, pp.1073-1076.

[21] Chatfield, A.T., Scholl, H.J., and Brajawidagda, U.: \#Sandy Tweets: Citizens' Co-Production of Time-Critical Information during an Unfolding Catastrophe. Proc. of the HICCS-47, 2014, pp. 1947-1957.

[22] Qu, Y., Huang, C., Zhang, P. and Zhang, J.: Microblogging after a major disaster in China: A case study of the 2010 Yushu Earthquake. CSCW, 2011, pp.25-34.

[23] Pelen, L., Vieweg, S., Liu, S.B., and Hughes, A. L.: Crisis in a networked world: Features of Computer-Mediated Communication in the April 16,2007, Virginia Tech Event. Social Science Computer Review, 2009, 27(4), pp. 467-480.

[24] Wilensky, H.: Twitter as a Navigator for Stranded Commuters during the Great East Japan Earthquake, Proc. of the 11th International ISCRAM Conf., 2014, pp.697-706.

[25] Turoff, M., Van De Walle, B., and Hiltz, S. R..: Emergency Response Information System Past, Present and Future, In: Van de Walle, B., Turoff, M., Hiltz, S.R. (eds.) Information Systems for Emergency Management, New York, 2010, pp.369-387.

[26] Kaigo Muneo : Social Media Usage During Disasters and Social Capital: Twitter and the Great East Japan Earthquake. Keio Communication Review, No.34, 2012, pp.19-35.

[27] Tanaka, Y., Sakamoto, Y., and Matsuka, T.: Toward a Social-Technological System that Inactivates False Rumors through the Critical Thinking of Crowds. Proc. of the HICCS-46, 2013, pp.649-658.

[28] Mobile Marketing Data (MMD) Report: Survey on social media use after the Great East Japan Earthquake.2011. http://mmd.up-date.ne.jp/news/detail.php?news_id=799

(in Japanese).

[29] Doan, S., Ho Vo, B., and Collier, N.: An analysis of Twitter messages in the 2011 Tohoku Earthquake. Lec. Notes of the Inst. for Comp. Sciences, Social Info. \& Telecom. Eng., Springer, 2012, pp.58-66.

[30] Schulze, K., Lorenz, D., Wenzel, B., and Voss, M.: Disaster Myths and their relevance for warning systems. Proceedings of ISCRAM, 2015.

[31] Gantt, P., and Gantt, R.: Disaster Psychology: Dispelling the Myths of Panic, Professional Safety, 2012, 57(8), pp. 4249.

[32] Li, H., Sakamoto, Y., Tanaka,Y. and Chen, R.: The Psychology Behind People's Decision to Forward DisasterRelated Tweets. Proc. Of the 18th Pacific Asia Conference on Information Systems, 2014.

[33] Li, H. and sakamoto, Y.:Re-tweet Count Matters:Social Influences on Sharing of Disaster-Related Tweets. Homeland Security \& Emergency Management,12(3),2015,pp.737-761. [34] Kowalski-Trakofler, K.M., Vaught, C., and Scharf, T.: Judgment and decision making under stress: An overview for emergency managers. Int. J. of Emergency Management. 1(3), 2003, pp. 278-289.

[35] Hiltz, S. R., Kushma, J., and Plotnick, L.: Use of Social Media by U.S. Public Sector Emergency Managers: Barriers and Wish Lists. Proc. of the 11th Int. ISCRAM Conf., 2014, pp. 697-706.

[36] Ruceuro, R, Araujo, R., and Zago, G.: How does social capital affect retweets?. In Proc. of $5^{\text {th }}$ Int. AAAI Conf. on Weblogs and Social Media. 2011, pp. 305-312.

[37] Starbird, K. and Palen, L.: Pass It On?: Retweeting in Mass Emergency. Proc. of the $7^{\text {th }}$ Int. ISCRAM Conf., 2010, pp. 1-10.

[38] Lee, K., Mahmud, J., Chen, J., Zhou, M. and Nichols, J.: Who will retweet this? Automatically Identifying and Engaging Strangers on Twitter to Spread Information. Proc. of IUI 2014. ACM, 2014, pp. 247-256.

[39] Shahab, Q., Terken, J. and Eggen, B.: Development of a questionnaire for identifying driver's personal values in driving. Proc. of the 5th International Conf. on Automotive User Interfaces and Interactive Vehicular Applications (AutomotiveUI,13), 2013, pp. 202-208.

[40] Choi, G. and Chung, H.: Applying the Technology Acceptance Model to So-cial Networking Sites (SNS): Impact of Subjective Norm and Social Capital on the Acceptance of SNS. Int. J. of Human-Computer Interaction, 29, 2013, pp. 619-628.

[41] Rashtian, H., Boshmaf, Y., Jaferian, P., and Beznosov, K.: To Befriend or Not? A Model of Friend Request Acceptance on Facebook. Symposium on Usable Privacy and Security (SOUPS), 2014, pp. 285-300. 
[42] Rossiter, J. R., and Lilien, G. L.: New "Brainstorming" Principles. Australian Journal of Management, 1994, 19(1).

[43] Nishioka, D., Murayama, Y., and Fujihara, Y.: Producing a questionnaire for a user survey on Anshin with information security for users without technical knowledge. Proc. of the HICCS-45, 2012, pp. 454-463.

[44] Sakaki, T., Toriumi, F., and Matsuo, Y.: Tweet trend analysis in an emergency situation, Proc. of the Special Workshop on Internet and Disasters (SWID '11), ACM Article 3, 2011.

[45] Field, A. : Discovering statistics using SPSS (Third edition). SAGE Publications, 2009.
[46] Cronbach, L. J.: Coefficient Alpha and the Internal Structure of Tests. Psychometrika, 1951, 16(3).

[47] Helsloot, I., and Ruitenberg, A.: Citizen response to disasters: A survey of literature and some practical implications. J of Contigencies and crisis management, 12(3), 2004, pp. 98-111.

[48] Shibutani, T.: Improvise news: A Sociological Study of Rumor. The Bobbs-Merrill Company, Inc. New York, 1966.

\section{Appendix. Descriptive statistics of all items in the questionnaire}

\begin{tabular}{|c|c|c|c|}
\hline No & Questions & Mean & S.D \\
\hline Q1 & I retweet because I believe true information is more than false information on Twitter. So, I should spread it. & 3.68 & 1.492 \\
\hline Q2 & I retweet the information that I know and have knowledge of it. & 3.82 & 1.540 \\
\hline Q3 & I read the information now, and it captured my interest. So, I retweet it. & 4.38 & 1.511 \\
\hline Q4 & There is no specific reason; I just follow my feeling (instinct) to retweet the information. & 3.10 & 1.523 \\
\hline Q5 & I retweet the information to attract the readers`attention to get famous. & 2.29 & 1.525 \\
\hline Q6 & I retweet because I feel excited to share about the unusual situation topic emerged in disaster situation & 3.00 & 1.632 \\
\hline Q7 & I retweet because I want to spread the warning information to people in my Twitter network. & 4.05 & 1.601 \\
\hline Q9 & I retweet because I believe my action could safe other people's life. & 3.96 & 1.606 \\
\hline Q10 & $\begin{array}{l}\text { I check the information if it is from a trusted source of information. For example, from televisions or newspaper, if } \\
\text { the content is same, then I will retweet it. }\end{array}$ & 4.56 & 1.562 \\
\hline Q11 & I know the information about the disaster in the disaster area, so I decided to retweet it. & 4.16 & 1.486 \\
\hline Q12 & I retweet because I could verify about the disaster situation while I am not in that disaster area. & 4.01 & 1.583 \\
\hline Q13 & I retweet because I think it is crucial (important) to share the information I read. & 4.68 & 1.595 \\
\hline Q14 & I retweet because I want to inform my followers who may not follow the specific Twitter account. & 4.01 & 1.616 \\
\hline Q16 & I do not know the retweet content in details. But if I think the information is important, I will retweet it. & 3.81 & 1.601 \\
\hline Q17 & I retweet because I want to allow my followers to add and tweet their opinion on this information. & 3.35 & 1.521 \\
\hline Q18 & I retweet because I want to get respond from disaster management professional who may read the information. & 3.14 & 1.530 \\
\hline Q19 & I retweet because I think it is good for every people to know about the disaster information. & 3.98 & 1.643 \\
\hline Q20 & I retweet because I feel Anshin (sense of security) after I saw the information received high number of retweet. & 3.41 & 1.566 \\
\hline Q21 & I will retweet if the one who retweeted the message has a good "follower" relation. & 3.83 & 1.534 \\
\hline Q22 & I retweet because I trust the informer (the people I follow). & 4.11 & 1.515 \\
\hline Q23 & I will retweet if the disaster information is related to my current situation. & 4.02 & 1.511 \\
\hline Q24 & I retweet because the information may relate to my followers situation. & 4.07 & 1.503 \\
\hline Q25 & $\begin{array}{l}\text { I retweet because by retweeting action, I could collect the disaster information that might be useful to my followers } \\
\text { and other people. }\end{array}$ & 4.03 & 1.528 \\
\hline Q26 & I retweet because information retweeted from Twitter is faster and updated than information from TV and news. & 3.94 & 1.546 \\
\hline Q27 & $\begin{array}{l}\text { I retweet because the information comes from trusted source and highly believable site. For example from } \\
\text { government website, NHK, CNN, BBC, NPR (local and foreign news). }\end{array}$ & 4.26 & 1.528 \\
\hline Q28 & I retweet because I can get detail information from local people rather than in news and TV. & 4.01 & 1.508 \\
\hline Q30 & I retweet the information which contains facts in it. & 3.40 & 1.544 \\
\hline Q31 & $\begin{array}{l}\text { I retweet the disaster information because I want to get advice on disaster preparation. For example, during flood, } \\
\text { what I should prepare and do, etc. }\end{array}$ & 3.33 & 1.547 \\
\hline Q32 & $\begin{array}{l}\text { I retweet because I can get early information from Twitter before I proceed with checking the safety status of my } \\
\text { friend and family thru telephone. }\end{array}$ & 3.84 & 1.612 \\
\hline Q33 & I retweet because retweet and hashtag(\#) functions helps and ease me to gather much information about the disaster. & 3.60 & 1.502 \\
\hline Q34 & I will retweet if the information was from reliable original author. & 3.78 & 1.521 \\
\hline Q35 & $\begin{array}{l}\text { I will retweet the information depends on the situation condition. For example, when there is the possibility that the } \\
\text { disaster will cause the damage to happen. (eg : information about landslide during heavy rain) }\end{array}$ & 4.00 & 1.476 \\
\hline Q36 & I will retweet if the information contains [Pls spread] written in it. & 3.55 & 1.562 \\
\hline Q37 & I will retweet if the information is for fun or joke. & 3.11 & 1.656 \\
\hline Q38 & I will retweet if the information is a positive thing. & 3.82 & 1.499 \\
\hline
\end{tabular}

